\title{
Liver Depurative Techniques: A Single Liver Transplantation Center Experience
}

\author{
J. Rodrigues ${ }^{a}$, S.G. Castro ${ }^{a}$, B. Moya ${ }^{a}$, P. Fortuna ${ }^{a}$, A. Martins ${ }^{a}$, J.P. Pereira ${ }^{b}$, L. Bento ${ }^{a}$, R. Perdigoto ${ }^{b}$, \\ E. Barroso ${ }^{b}$, and P. Marcelino ${ }^{a, *}$

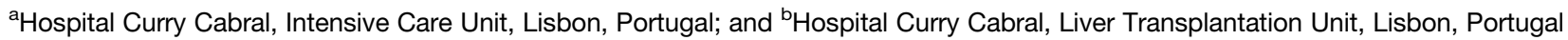

\begin{abstract}
Background. In a liver transplant (LT) center, treatments with Prometheus were evaluated. The main outcome considered was 1 and 6 months survival.

Methods. During the study period, 74 patients underwent treatment with Prometheus; 64 were enrolled, with a mean age of $51 \pm 13$ years; 47 men underwent 212 treatments (mean, 3.02 per patient). The parameters evaluated were age, sex, laboratorial (liver enzymes, ammonia) and clinical (model for end-stage liver disease and Child-Turcotte-Pugh score) data.

Results. Death was verified in 23 patients $(35.9 \%)$ during the hospitalization period, 20 patients $(31.3 \%)$ were submitted to liver transplantation, and 21 were discharged. LT was performed in 4 patients with acute liver failure (ALF, 23.7\%), in 7 patients with acute on chronic liver failure (AoCLF, 43.7\%), and in 6 patients with liver disease after LT (30\%). Seven patients who underwent LT died (35\%). In the multivariate analysis, older age $(P=.015)$, higher international normalized ratio (INR) $(P=.019)$, and acute liver failure $(P=.039)$ were independently associated with an adverse 1-month clinical outcome. On the other hand, older age $(P=.011)$ and acute kidney injury $(P=.031)$ at presentation were both related to worse 6-month outcome. For patients with ALF and AoCLF we did not observe the same differences.

Conclusions. In this cohort, older age was the most important parameter defining 1- and 6-month survival, although higher INR and presence of ALF were important for 1-month survival and AKI for 6-month survival. No difference was observed between patients who underwent LT or did not have LT.
\end{abstract}

$\mathbf{L}$ IVER failure is a critical illness characterized by jaundice, porto-systemic encephalopathy syndrome, coagulopathy, and high mortality rates. The condition can be precipitated in patients with or without chronic liver disease and has several possible causes, including virus, toxins, drugs, or metabolic stress [1,2].

Some patients recover spontaneously, but liver transplantation (LT) achieves approximately $90 \%$ of cure rates [1] and has become the therapy of choice for these patients. Because of an organ shortage, effective therapies that allow managing periods of decompensation or to bridge to LT transplantation are still needed [3]. For this purpose, artificial extracorporeal liver support systems have been created, including the Molecular Adsorbents Recirculating System (MARS) and Fractioned Plasma Separation and
Adsorption (FPSA - Prometheus), with the ability to remove albumin-bound toxins.

These systems are promising in patients with acute-onchronic liver failure (AoCLF) and acute liver failure (ALF), but there is lack of robust randomized, clinical trials as far as outcomes and effective bridging to transplant are concerned.

At the leading LT center in Portugal, a high number of patients have been treated with both techniques, mostly Prometheus, for the past 6 years. The aims of this study were to assess the clinical experience with liver depurative

*Address correspondence to Paulo Marcelino, Hospital Curry Cabral, Intensive Care Unit, Rua da Beneficência, 8 1069-166 Lisbon, Portugal. E-mail: jp.azevedo.rodrigues@gmail.com

(c) 2015 by Elsevier Inc. All rights reserved. 360 Park Avenue South, New York, NY 10010-1710 
techniques and to highlight the groups of patients who might benefit the most from this treatment, considering major clinical outcomes and survival.

\section{MATERIALS AND METHODS}

The study was retrospective, and data were collected from June 2008 to December 2012. All patients admitted to Curry Cabral Hospital who were submitted to Prometheus were included. Exclusion criteria were incomplete data and isolated incomplete session.

The data collected consisted of demographic information (age, sex), laboratorial data before the first treatment (aspartate transaminase [AST]; alanine transaminase [ALT]; gamma-glutamyltransferase [GGT]; alkaline phosphatase [ALP]; international normalized ratio [INR], ammonia; and total bilirubin, collected before and after Prometheus treatment), and model for end-stage liver disease (MELD) and Child-Turcotte-Pugh (CTP) score, also considered before the first treatment. Comorbidities were also taken into account, namely acute kidney injury (AKI) and presence of any dialysis technique, presence of mechanical ventilation, and presence of continuous infusion of vasopressors, also before the first treatment. Other data retrieved included number of treatments per patient and technical complications, namely, hypotension (leading to further intervention such as fluid or vasopressor administration), filter coagulation, and other complications recorded. Patients were divided into 5 groups, according to the indications for Prometheus treatment: ALF, AoCLF, posttransplant liver disorders (rejection and with no mention of rejection), cholestatic liver diseases not included in the previous groups, and hepatic encephalopathy syndrome (HES). ALF was defined as an INR $\geq 1.5$ and any degree of mental alteration (HES) in a patient without pre-existing cirrhosis and with an illness with less than 26-week duration. AoCLF was defined as 2 organ failures or a single kidney failure in a patient with pre-existing cirrhosis. The need for treatment was decided by a team that included LT surgeons, hepatologists, and intensivists on a daily basis and considering the patient's evolution. This team was also was responsible for the decision on LT.

The major results considered were hospital discharge, transplantation, or inpatient death, and 1-month and 6-month survival rates were regarded as the major outcomes.

The Prometheus treatment was performed under an established protocol and performed by trained professionals from Fresenius. The protocol included a 6-hour treatment with the filters provided by Fresenius, with the use of a continuous infusion of nonfractionated heparin on a rhythm of $500 \mathrm{IU}$ per hour. The need for ultrafiltration or dialysis was evaluated case by case.

Continuous variables are expressed as an average and standard deviation. Categorical variables are presented as categories with percentages. For comparative analysis, parametric (Student $t$ test for numeric variables, after confirmation of the normal distribution of data by the Kolmogorov-Smirnov test) and non-parametric tests ( $\chi^{2}$ test or Fisher exact test for categorical variables) were used. To establish dependence between variables, logistic regression analysis was performed (backward-LR), with the use of variables that were significant on univariate analysis. A goodness-of-fit test (HosmerLemeshow) was used to assess the fit of the logistic regression model. The variables identified in the univariate analysis were used in the multivariate regression model. The dependent variables considered were the survival at 1 and 6 months; the independent variables were those evaluated (clinical, laboratorial, and demographic variables). For the most significant results, a receiver operator characteristics (ROC) curve was generated, and the area
Table 1. Indications for Prometheus Treatment

\begin{tabular}{lc}
\hline \multicolumn{1}{c}{ Indication } & $\begin{array}{c}\text { Total }(\mathrm{n}=64) \\
\mathrm{n}(\%)\end{array}$ \\
\hline Acute liver failure & $17(26.6 \%)$ \\
Acute-on-chronic liver failure & $16(25 \%)$ \\
Post-transplant & $20(31.3 \%)$ \\
Rejection & 6 \\
$\quad$ No rejection mentioned & 14 \\
Cholestatic liver disease & $16(25 \%)$ \\
Hepatic encephalopathy syndrome & $19(29.7 \%)$ \\
\hline
\end{tabular}

under the curve was considered. A value of $P<.05$ was considered statistically significant. A comparative analysis between patients with an adverse and favorable clinical outcome was performed in the whole cohort as well as for patients with ALF and AoCLF. Statistical analysis was performed with the use of SPSS 19.0 (Statistical Package for Social Sciences, SPSS, Inc., Chicago, Ill, United States).

\section{RESULTS}

During the study period, 74 patients underwent treatment with Prometheus, and 64 met the inclusion criteria, who underwent 212 treatments (mean, 3.02 per patient; range, 1-11). This cohort presented a mean age of $51 \pm 13$ years; 47 were men. The patient groups considered, regarding indications to Prometheus, are presented in Table 1.

AKI was the most common comorbidity $(n=27,42 \%)$. Dialysis was present 11 patients, mechanical ventilation in 6 , and vasopressor support in 4. Laboratorial data are presented in Table 2. Mean bilirubin level was $27.3 \pm 12.7$, which decreased $13.61 \pm 10.09$ after Prometheus sessions. The laboratorial and clinical data for the whole cohort is presented in Table 2, and in Table 3 these data are presented separately for patients with ALF and AoCLF.

Death was verified in 23 patients $(35.9 \%)$ during the hospitalization period, 20 patients $(31.3 \%)$ were submitted to liver transplantation, and 21 were discharged. LT was performed in 4 patients with ALF $(23.7 \%)$, in 7 patients with AoCLF $(43.7 \%)$ and in 6 patients with liver disease after LT (30\%), and in other patients with other underlying

Table 2. Laboratory Evaluation Before First Treatment of the Whole Cohort $(n=64)$

\begin{tabular}{lc}
\hline & Mean $( \pm \mathrm{SD})$ \\
\hline MELD & $29.4( \pm 9.2)$ \\
CTP & $10.6( \pm 2)$ \\
AST $(\mathrm{U} / \mathrm{L})$ & $449( \pm 886)$ \\
ALT $(\mathrm{U} / \mathrm{L})$ & $427( \pm 842)$ \\
GGT $(\mathrm{U} / \mathrm{L})$ & $280( \pm 1032)$ \\
ALP $(\mathrm{U} / \mathrm{L})$ & $334( \pm 424)$ \\
Total bilirubin $(\mathrm{mg} / \mathrm{dL})$ & $27.3( \pm 12.7)$ \\
Arterial ammonia $(\mu \mathrm{g} / \mathrm{dL})$ & $81.2( \pm 47.8)$ \\
INR & $2.3( \pm 1.9)$ \\
\hline
\end{tabular}

Abbreviations: AST, aspartate transaminase; ALT, alanine transaminase; GGT, gamma-glutamyltransferase; ALP, alkaline phosphatase; INR, international normalized ratio. 
Table 3. Laboratory Evaluation Before First Treatment of Patients With ALF and AoCLF

\begin{tabular}{lcc}
\hline & $\begin{array}{c}\text { ALF } \\
(\mathrm{n}=17)\end{array}$ & $\begin{array}{c}\text { AoCLF } \\
(\mathrm{n}=16)\end{array}$ \\
\hline MELD (mean $\pm \mathrm{SD})$ & $27 \pm 9.2$ & $37.1 \pm 7.7$ \\
CTP (mean \pm SD) & $10.3 \pm 2.3$ & $12.3 \pm 1.3$ \\
AST (U/L, mean \pm SD) & $684 \pm 1258$ & $196 \pm 177$ \\
ALT (U/L, mean \pm SD) & $752 \pm 1352$ & $194 \pm 220$ \\
GGT (U/L, mean \pm SD) & $164 \pm 170$ & $195 \pm 186$ \\
ALP (U/L, mean \pm SD) & $267 \pm 205$ & $185 \pm 114$ \\
Total bilirubin (mg/dL, mean \pm SD) & $28.8 \pm 14.4$ & $31.1 \pm 13.4$ \\
Arterial ammonia $(\mu \mathrm{g} / \mathrm{dL}$, mean $\pm \mathrm{SD})$ & $86 \pm 37$ & $99 \pm 66$ \\
INR (mean \pm SD) & $1.9 \pm 0.9$ & $2.2 \pm 0.5$ \\
\hline
\end{tabular}

Abbreviations: AST, aspartate transaminase; ALT, alanine transaminase GGT, gamma-glutamyltransferase; ALP, alkaline phosphatase; INR, international normalized ratio.

pathologies. Seven patients who underwent LT died (35\%), not reaching statistical significance.

The most common complication was hypotension $(\mathrm{n}=21$, $32.8 \%$ ), with only a minority requiring initiation of vasopressor support $(\mathrm{n}=6,9.4 \%)$, or dose adjustment $(\mathrm{n}=3$, $4.7 \%$ ). Filter clotting was observed in 5 patients $(7.8 \%)$. Other complications included agitation $(\mathrm{n}=2)$, myoclonus $(\mathrm{n}=1)$, hypertension $(\mathrm{n}=1)$, and nausea $(\mathrm{n}=1)$.

We were not able to record 1- and 6-month survival rates from 8 patients because they were transferred to other health care units. Of the remaining 56 patients, $33(58.9 \%)$ were alive at 1 month and $23(35.9 \%)$ at the 6th month. By univariate analysis, we observed that younger age, cholestatic liver disease, and lower CTP score were related to improvement in 1- and 6-month survival rates. Higher INR $(P=.020)$ and ALF $(P=.025)$ were negatively associated with 1-month survival rate and the presence of AKI $(P=.013)$, and higher MELD score $(P=.026)$ with worse 6-month outcome. These data are presented in Table 3. In the multivariate analysis, older age $(P=.015)$, higher INR $(P=.019)$, and ALF $(P=.039)$ were independently associated with an adverse 1-month clinical outcome. On the other hand, older age $(P=.011)$ and AKI $(P=.031)$ at presentation were both related to worse 6 -month outcome (Table 4 and Table 5). The ROC curves for 1-month survival and INR and age and 6-month survival are presented in Fig 1 and Fig 2, respectively. For patients with ALF and AoCLF we did not observe the same differences.

\section{DISCUSSION}

In this study, a series of 212 treatments in 64 patients with Prometheus, we observed that older age, higher INR, and ALF were independently associated with an adverse 1-month clinical outcome. On the other hand, older age and AKI at presentation were both related to worse 6-month outcome. Nonetheless, these data concern all patients enrolled, and we could not find any distinction between patients with ALF and AoCLF. We should stress that the presented data are quite significant regarding the number of treatments and patients described. The significant data that
Table 4. Multivariate Analysis of Factors Associated With One-Month and Six-Month Outcomes

\begin{tabular}{|c|c|c|}
\hline & $\begin{array}{c}\text { One-Month Survival } \\
\quad P \text { Value }\end{array}$ & $\begin{array}{c}\text { Six-Month Survival } \\
P \text { Value }\end{array}$ \\
\hline Age & $\begin{array}{l}P=.020 \\
\quad \mathrm{RC}:-0.064\end{array}$ & $\begin{array}{l}P=.006 \\
\quad \mathrm{RC}:-0.079\end{array}$ \\
\hline Sex & $P=.548$ & $P=.548$ \\
\hline Cholestatic liver disease & $\begin{array}{l}P=.045 \\
\text { RC: } 1.658\end{array}$ & $\begin{array}{l}P=.06 \\
\quad \text { RC: } 2.040\end{array}$ \\
\hline ALF & $\begin{array}{l}P=.025 ; \\
\quad \mathrm{RC}:-1.674\end{array}$ & $P=.101$ \\
\hline Post-transplant disorder & $P=.213$ & $P=.656$ \\
\hline $\mathrm{HE}$ & $P=.304$ & $P=.645$ \\
\hline AoCLF & $P=.607$ & $P=.478$ \\
\hline $\begin{array}{l}\text { Total bilirubin } \\
\text { (pre-Prometheus) }\end{array}$ & $P=.454$ & $P=.471$ \\
\hline $\begin{array}{l}\text { Bilirubin fall } \\
\text { (post-Prometheus) }\end{array}$ & $P=.295$ & $P=.375$ \\
\hline AKI & $P=.861$ & $\begin{array}{l}P=.013 ; \\
\quad \text { RC: }-1.472\end{array}$ \\
\hline MELD & $P=.269$ & $\begin{array}{l}P=.026 \\
\quad \text { RC: }-0.361\end{array}$ \\
\hline CTP & $\begin{array}{l}P=.037 \\
\quad \mathrm{RC}:-0.332\end{array}$ & $\begin{array}{l}P=.039 \\
\quad \text { RC: }-0.321\end{array}$ \\
\hline INR & $\begin{array}{l}P=.020 \\
\quad \mathrm{RC}-0.812\end{array}$ & $P=.076$ \\
\hline Ammonia & $P=.123$ & $P=.141$ \\
\hline ALT & $P=.581$ & $P=.774$ \\
\hline Hypotension (TC) & $P=.480$ & $P=.211$ \\
\hline
\end{tabular}

Abbreviations: ALF, acute liver failure; HE, hepatic encephalopathy; AoCLF, acute-on-chronic liver failure; AKI, acute kidney injury; MELD, model for end-stage liver disease; CTP, Child-Turcotte-Pugh score; INR, international normalized ratio; ALT, alanine transaminase; TC, technical complication; RC, regression coefficient.

we describe are generally in accordance with the literature, but, in specific patient groups, we could not find those who could benefit the most with Prometheus. Only the general data can be taken into account, and they deserve attention. Regardless of the clinical condition, in this cohort, clinical data were more important to the outcome than the specific clinical condition, namely ALF or AoCLF.

The biological and gross laboratorial effects of liver depurative techniques are well described. Smaller studies proved the reduction of bilirubin [4-10], bile acids [11],

Table 5. Multivariate Analysis of Factors Associated With One-Month and Six-Month Outcomes

\begin{tabular}{lll}
\hline & \multicolumn{1}{c}{$\begin{array}{c}\text { One-Month Survival } \\
P \text { Value }\end{array}$} & \multicolumn{1}{c}{$\begin{array}{c}\text { Six-Month Survival } \\
P \text { Value }\end{array}$} \\
\hline Age & $P=.015 ;$ OR: 0.930 & $P=.011 ;$ OR: 0.929 \\
& $\mathrm{Cl}:-0.133 ;-0.013$ & $\mathrm{Cl}:-0.132 ;-0.016$ \\
ALF & $P=.039 ;$ OR: 0.166 & $\mathrm{~N} / \mathrm{A}$ \\
& $\mathrm{Cl}:-3.538 ;-0.054$ & \\
AKI & $\mathrm{N} / \mathrm{A}$ & $P=.031 ;$ OR: 0.253 \\
& & $\mathrm{Cl}:-2.635 ;-0.097$ \\
INR & $P=.019 ;$ OR: 0.378 & $\mathrm{~N} / \mathrm{A}$ \\
& $\mathrm{Cl}:-1.802 ;-0.013$ & \\
\hline
\end{tabular}

Abbreviations: ALF, acute liver failure; $\mathrm{AKI}$, acute kidney injury; $\mathrm{Cl}$, confidence interval; INR, international normalized ratio; OR, odds ratio; N/A, not applicable. 


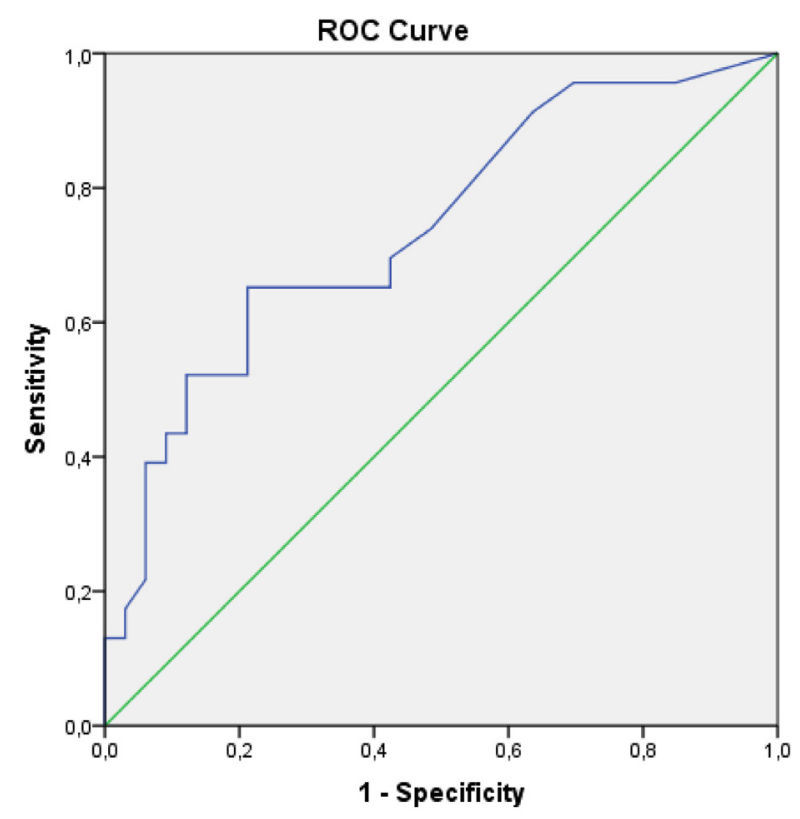

Diagonal segments are produced by ties.

Fig 1. Receiver operator characteristics for 1-month survival and INR (area under the curve, 0.739).

amino acids [12], and ammonia [9], with conflicting results relating inflammation markers such as tumor necrosis factor-alpha $[10,13,14]$, and interleukin-6 [10,14]. MARS was described in the improvement of hemodynamic parameters such as mean arterial pressure in 2 randomized,

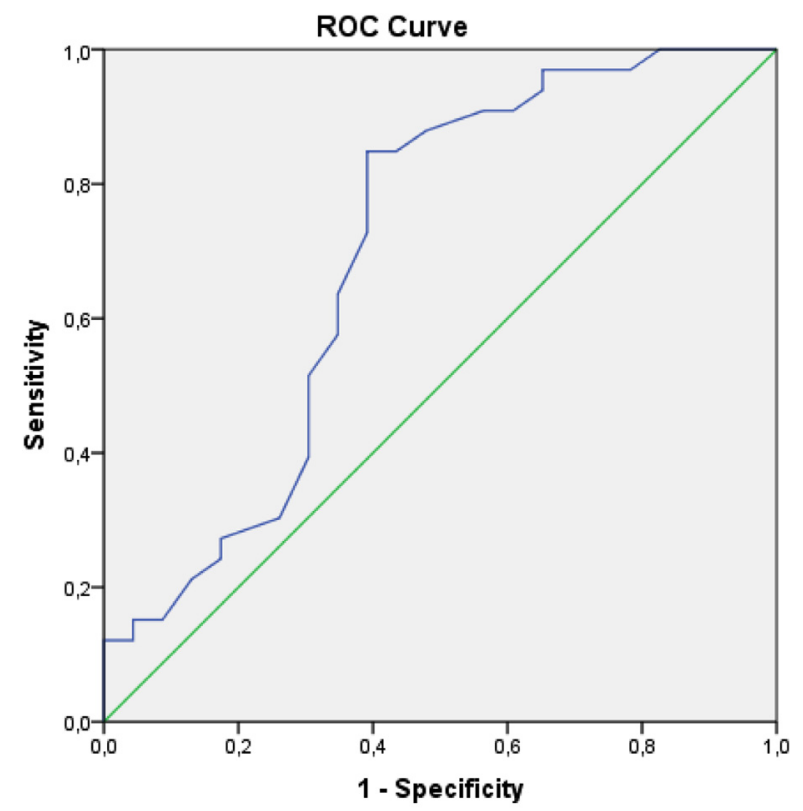

Diagonal segments are produced by ties.

Fig 2. Receiver operator characteristics for 6-month survival and age (area under the curve, 0.698). controlled studies, although no clinical outcome could be associated $[7,15]$.

Larger clinical studies aiming at clinical outcomes are still scarce. The studied biological effects, such as the observed decrease in serum bilirubin, did not affect the 1-month or 6-month survival. Yet, in this cohort, hypotension and need to increase vasopressor support, markers of systemic inflammation, were not frequent.

One of the largest randomized, controlled clinical trials thus far (RELIEF trial) showed that MARS reduces organ dysfunction but has no impact on 28- and 90-day survival without transplantation on patients with AoCLF [16]. Similarly, a prospective, randomized, controlled, parallelgroup multicenter trial reported no increase in the probability of survival of patients with AoCLF who were treated with the Prometheus system when compared with standard medical therapy (SMT); however, patients with a MELD score greater than 30 treated with Prometheus had a statistically significant better survival rate compared with that of patients treated with SMT only [17]. Another randomized, controlled trial with 102 patients with ALF found no statistical significance in the 6-month survival rate between SMT and MARS [18]. Presented data could not reproduce the benefit for higher MELD, but higher INR and the presence of ALF influenced adversely the 1-month survival, and older age was the sole parameter adversely affecting both 1-month and 6-month survival. Nonetheless, in the univariate analysis, both MELD and CTP could be related to survival but lose statistical significance in the multivariate regression model.

A retrospective study that included 52 patients with ALF treated with Prometheus showed $68 \%$ mortality in patients who did not undergo LT; among the 28 patients who survived, 22 underwent LT. The authors suggested that Prometheus should be reserved as a bridging therapy [6]. In the studied cohort, we did not observe this fact, but patients with AoCLF more frequently underwent LT (43.7\%), although the after-LT survival was not affected.

Another study comparing Prometheus with MARS in patients with AoCLF also found that both led to improvement and transplantation in $70 \%$ of the patients and that the treatment appeared to be pointless in primary nonfunctional and secondary liver failure cases [19]. Likewise, Oppert et al [4] observed global survival rate of $26 \%$ in patients with ALF and AoCLF submitted to Prometheus, with a higher survival rate among ALF patients. These data could not be reproduced in the present study; we did not find any difference in 6-month survival for patients who underwent LT and those who did not undergo LT.

\section{Study Limitations}

In our data, we could not compare different liver depurative techniques. The data only referred to Prometheus, and they should be considered in that specific treatment. It is difficult to conduct properly randomized studies for comparison of these most widely used techniques. Also, beside technical 
issues, relevant clinical outcomes or different biological effects are yet to be described for these techniques.

\section{CONCLUSIONS}

In 64 patients who underwent 212 treatments with Prometheus, we found that older age was related to 1-month and 6-month survival. Higher INR $(P=.019)$ and acute liver failure $(P=.039)$ were independently associated with an adverse 1 -month clinical, and AKI $(P=.031)$ at presentation was related to worse 6-month outcome. For patients with ALF and AoCLF we did not observe the same differences. Severity scores at presentation (MELD and CTP) were related to 1- and 6-month survival only in the univariate analysis. We concluded that in the group of patients studied, age was the most important parameter defining 1- and 6-month survival. LT was not related to a better survival in this cohort.

\section{REFERENCES}

[1] Kjaergard LL, Liu J, Als-Nielsen B, Gluud C. Artificial and bioartificial support systems for acute and acute-on-chronic liver failure: a systematic review. JAMA 2003;289:217-22.

[2] Bernal W, Wendon J. Acute liver failure. N Engl J Med 2013;369:2525-34.

[3] Rifai K, Manns MP. Review article: clinical experience with Prometheus. Therapeut Apheresis Dial 2006;10:132-7.

[4] Oppert M, Rademacher S, Petrasch K, Jörres A. Extracorporeal liver support therapy with prometheus in patients with liver failure in the intensive care unit. Therapeut Apheresis Dial 2009;13: 426-30.

[5] Santoro A, Faenza S, Mancini E, et al. Prometheus system: a technological support in liver failure. Transplant Proc 2006;38: 1078-82.

[6] Grodzicki M, Kotulski M, Leonowicz D, et al. Results of treatment of acute liver failure patients with use of the Prometheus FPSA system. Transplant Proc 2009;41:3079-81.

[7] Laleman W, Wilmer A, Evenepoel P, et al. Effect of the molecular adsorbent recirculating system and Prometheus devices on systemic haemodynamics and vasoactive agents in patients with acute-on-chronic alcoholic liver failure. Crit Care 2006;10:R108.

[8] Rifai K, Ernst T, Kretschmer U, et al. Prometheus: a new extracorporeal system for the treatment of liver failure. J Hepatol 2003;39:984-90.

[9] Skwarek A, Grodzicki M, Nyckowski P, et al. The use Prometheus FPSA system in the treatment of acute liver failure: preliminary results. Transplant Proc 2006;38:209-11.

[10] Rifai K, Ernst T, Kretschmer U, et al. Removal selectivity of Prometheus: a new extracorporeal liver support device. World J Gastroenterol 2006;12:940-4.

[11] Stadlbauer V, Krisper P, Beuers U, et al. Removal of bile acids by two different extracorporeal liver support systems in acuteon-chronic liver failure. ASAIO J 2007;53:187-93.

[12] Pares A, Deulofeu RN, Cisneros L, et al. Albumin dialysis improves hepatic encephalopathy and decreases circulating phenolic aromatic amino acids in patients with alcoholic hepatitis and severe liver failure. Crit Care 2009;13:1.

[13] Rocen M, Kieslichova E, Merta D, et al. The effect of Prometheus device on laboratory markers of inflammation and tissue regeneration in acute liver failure management. Transplant Proc 2010;42:3606-11.

[14] Stadlbauer V, Krisper P, Aigner R, et al. Effect of extracorporeal liver support by MARS and Prometheus on serum cytokines in acute-on-chronic liver failure. Crit Care 2006;10:R169.

[15] Dethloff T, Tofteng F, Frederiksen H-J, et al. Effect of Prometheus liver assist system on systemic hemodynamics in patients with cirrhosis: a randomized controlled study. World J Gastroenterol 2008;14:2065-71.

[16] Bañares R, Nevens F, Larsen FS, et al. Extracorporeal Albumin dialysis with the molecular adsorbent recirculating system in acute-on-chronic liver failure: the RELIEF trial. Hepatology 2013;57:1153-62.

[17] Kribben A, Gerken G, Haag S, et al. Effects of fractionated plasma separation and adsorption on survival in patients with acuteon-chronic liver failure. Gastroenterology 2012;142:782-9.

[18] Saliba F, Camus C, Durand F, et al. Albumin dialysis with a noncell artificial liver support device in patients with acute liver failure: a randomized, controlled trial. Ann Intern Med 2013;159: $522-31$.

[19] Faenza S, Baraldi O, Bernardi M, et al. MARS and Prometheus: our clinical experience in acute chronic liver failure. Transplant Proc 2008;40:1169-71. 